\title{
Health Literacy: Pure and Simple
}

\author{
Koenig VE* \\ Occupational Therapy, Touro College, USA
}

*Corresponding author: Virginia E Koenig, OTD, MSA, OTR, Occupational therapy,

Touro College, 320 West 31st Street New York, NY 10001, USA, Tel: 516-446-0082;

Email: Virginia.crippen@touro.edu

\section{Editorial}

Volume 1 Issue 2

Received Date: November 12, 2018

Published Date: November 21, 2018

DOI: $10.23880 /$ aphot-16000109
Keywords: Health literacy; Occupational and physical therapists; Health care; Toolkit

Abbreviations: AHRQ: Agency for Healthcare Research and Quality; AMA: American Medical Association; IOM: Institute of Medicine; REALM-: Rapid Estimate of Adult Literacy in Medicine; SAHL-S/E: Short Assessment of Health Literacy, Spanish/English.

\section{Editorial}

Policy priorities and growing evidence indicates occupational and physical therapists need to consider their clients' levels of health literacy if they are to provide effective, client-centered treatment [1]. Limited health literacy is associated with numerous adverse healthrelated outcomes, including higher overall health costs, excessive hospitalizations, and increased mortality rates [2]. Low health literacy is a threat to the health and wellbeing of individuals on national and global levels. Incorporating health literacy initiatives into clinical practice is also vital for the overall improvement and efficiency of health care systems. Both health literacy and rehabilitation requires the interrelationship of capacities, functioning, and client participation for successful outcomes; therefore, they are interconnected. Nevertheless, a number of studies indicate that health care professionals, such as occupational and physical therapists, have a difficult time identifying patients who may be low-health literate. Consequently, occupational and physical therapists must consider their client's health literacy levels if they are to provide effective clientspecific services.

\section{What is Health Literacy?}

Health literacy is a person's ability to obtain, process, and understand rudimentary health information and services adequately enough to make appropriate health care decisions [3]. Unlike general literacy, health literacy is considered a more dynamic and context-reliant ability. Literature reviews funded by the Agency for Healthcare Research and Quality (AHRQ), a Division of the United States (U.S.) Department of Health and Human Services, show that approximately one-third of U.S. adults have limited health literacy, as measured by their ability to understand health information from written sources. That figure rises to 41 percent for adults aged 65-75, and 70 percent for those older than 75 [4]. Additionally, twothirds of older adults live with chronic conditions such as diabetes, hypertension, and depression that can further restrict their ability to participate in everyday activities. Because chronic health conditions have no cure and are often medically complex, patients must learn to selfmanage their chronic conditions via lifestyle and behavioral changes, which can help minimize their adverse effects on everyday roles, routines, and habits [5]. Successful self-management of chronic diseases necessitates health-literate savvy clinicians who can help their patients clearly understand recommended medical routines, and navigate a complex health care system in order to obtain and effectively respond to rehabilitation procedures, processes, and services.

\section{How Can Occupational and Physical Therapists Address Health Literacy?}

A highlighted aspect of the National Action Plan to Improve Health Literacy is the need for concerted efforts by all stakeholders to improve and disseminate health literacy education to health care professionals [5]. Reports issued by the American Medical Association (AMA) and Institute of Medicine (IOM) also call for greater efforts to educate stakeholders, such as occupational and physical therapists, about health literacy and effective low-health literacy communication techniques [6]. 


\section{Annals of Physiotherapy \& Occupational Therapy}

In response to the alarming prevalence of low health literacy and its influence on U.S. health care systems, services, and outcomes, the AHRQ commissioned the Health Literacy Universal Precautions Toolkit, $2^{\text {nd }}$ edition (the Toolkit). A universal precautions approach to health literacy supposes that all patients experience difficulties understanding health information; therefore, any instructions provided to patients should be communicated in a manner that anyone can understand. For example, patient education material is often written above the reading level of most adults. The average U.S. adults' reading skills is at approximately a $7^{\text {th }}$ or $8^{\text {th }}$ grade level. Consequently, universal precautions prescribe that written educational material be provided to all patients at a fifth grade reading level. This helps to ensure that information provided to patients are easy to understand and recommended medical regimens are successfully followed-through [7]. The Toolkit is an online resource that can help occupational and physical therapists actively adopt a systematic approach to reducing complexities associated with low heath literacy and rehabilitation services. Routine use of the Toolkit also helps to ensure that patients can succeed in a dynamic physical medicine and rehabilitation environment. Occupational and physical therapists who adopt a universal precautions approach to health literacy can help reduce the complexities associated with health care, improve patient understanding of pertinent health information, and increase support for all patients, regardless of their health literacy level.

In addition to the Toolkit, other AHRQ-funded health literacy tools, such as the Rapid Estimate of Adult Literacy in Medicine-Short Form (REALM-SF) and the Short Assessment of Health Literacy, Spanish/English (SAHLS/E) are also available online to health care professionals. While these AHRQ-supported tools were designed to measure individuals' reading comprehension in a medical context, each instrument has strengths, but also limitations ranging from inaccurately measuring comprehension to being too time-consuming to implement [8]. Because of the high prevalence of lowhealth literacy ,ensuring patients' understanding of health information using universal precautions appears to be a pragmatic and reasonable alternative to individual screening. Furthermore, screening for health literacy is a selective, discriminatory process; therefore, any level of health literate care that falls short of universal precautions potentially excludes many who are at risk for low health literacy [9]. For these reasons, it is emphasized that AHRQ-supported health literacy measurement tools be used by occupational and physical therapists for research, program planning, and quality assurance purposes and not for routine clinical use.

Evidence indicates that physicians and non-physician health care professionals benefit from health literacy training. The Toolkit can provide occupational and physical therapists with evidence-based guidance on how to address low health literacy in their clinical practice. Research also signifies that curricula aimed at increasing health care professionals' awareness of health literacy, in addition to obtaining knowledge of the tools, competencies, and strategies that can promote health literacy in professional practice, potentially improves overall patient treatment and outcomes [10]. Furthermore, it is imperative that in-depth health literacy research continues on institutional, national, and global levels so that all stakeholders can base their actions and plans of care on the best possible evidence. Simply, recruitment of the Toolkit and other AHRQ-funded health literacy tools can facilitate occupational justice (engagement in individual-specific activities and tasks in a chosen environment) on micro, meso, and macro societal levels.

\section{Health Literacy: The Time is now!}

In its purest sense, health literacy is an issue of ethics and equity and is fundamental to reducing health care disparities. As the U.S. population becomes progressively diverse, occupational and physical therapists will become increasingly challenged to understand the effect health literacy has on the occupational performance of varied patient populations [11]. In order for low-health literate patients to respond effectively to health information provided to them throughout the rehabilitation process, it is paramount that occupational and physical therapists be sensitive to their patients' individual needs. The increased use of health literacy universal precautions in routine clinical practice, and other AHRQ-supported health literacy tools for research and program planning will help to improve the care and outcomes for at-risk patients and populations across the lifespan.

\section{References}

1. Levasseur M, Carrier A (2012) Integrating health literacy into occupational therapy: findings from a scoping review. Scand J Occup Ther 19(4): 305-314.

2. Zanchetta M, Maheu C, Fontaine C, Salvador-Watts L, Wong N et al. (2014) Awakening professionals. 


\section{Annals of Physiotherapy \& Occupational Therapy}

3. critical awareness of health literacy issues within a francophone linguistic minority population in Ontario. Chronic Disease and Injuries in Canada 34(4): 236-247.

4. Kobayashi LC, Wardle J, Wolf MS (2014) Aging and Functional Health Literacy: A Systematic Review and Meta-Analysis. The Journals of Gerontology Series B: Psychological Sciences and Social Sciences 71(3): 445-457.

5. Berkman ND, Sheridan SL, Donahue KE, Halpern DJ (2011) Low health literacy and health outcomes: an updated systematic review. Ann Intern Med 155(2): 97-107.

6. Geboers B, Brainard JS, Loke YK, Jansen CJ (2015) The association of health literacy with adherence in older adults, and its role in interventions: a systematic meta-review. BMC Public Health 15: 903.

7. DeWalt DA, Broucksou KA, Hawk V, Brach C (2011) Developing and testing the health literacy universal precautions toolkit. Nurs Outlook 59(2): 85-94.
8. Green JA, Gonzaga AM, Cohen ED, Spagnoletti CL (2014) Addressing health literacy through clear communication: a training program for internal medicine residents. Patient Educ Couns 95(1): 76-82.

9. AHRQ Health Literacy Universal Precautions Toolkit (2015) AHRQ-Agency for Healthcare Research and Quality.

10. AHRQ Heath Literacy Measurement Tools Revised (2016) AHRQ-Agency for Healthcare Research and Quality.

11. Coleman C (2011) Teaching health care professionals about health literacy: A review of the literature. Nurs Outlook 59(2): 70-78.

12. Liang L, Brach C (2017) Health literacy universal precautions are still a distant dream: analysis of u.s. data on health literate practices. Health Lit Res Pract 1(4): e216-230. 